\title{
Broadband tunable liquid crystal terahertz waveplates driven with porous graphene electrodes
}

\author{
Lei Wang ${ }^{1, *}$, Xiao-Wen Lin ${ }^{1, *}$, Wei Hu ${ }^{1}$, Guang-Hao Shao ${ }^{1}$, Peng Chen ${ }^{1}$, Lan-Ju Liang ${ }^{2}$, Biao-Bing Jin ${ }^{2}$, \\ Pei-Heng $\mathrm{Wu}^{2}$, Hao Qian ${ }^{3}$, Yi-Nong $\mathrm{Lu}^{3}$, Xiao Liang ${ }^{4}$, Zhi-Gang Zheng ${ }^{1}$ and Yan-Qing Lu ${ }^{1}$
}

Versatile devices, especially tunable ones, for terahertz imaging, sensing and high-speed communication, are in high demand. Liquid crystal based components are perfect candidates in the optical range; however, they encounter significant challenges in the terahertz band, particularly the lack of highly transparent electrodes and the drawbacks induced by a thick cell. Here, a strategy to overcome all these challenges is proposed: Few-layer porous graphene is employed as an electrode with a transmittance of more than $98 \%$. A subwavelength metal wire grid is utilized as an integrated high-efficiency electrode and polarizer. The homogeneous alignment of a high-birefringence liquid crystal is implemented on both frail electrodes via a non-contact photo-alignment technique. A tunable terahertz waveplate is thus obtained. Its polarization evolution is directly demonstrated. Furthermore, quarter-wave plates that are electrically controllable over the entire testing range are achieved by stacking two cells. The proposed solution may pave a simple and bright road toward the development of various liquid crystal terahertz apparatuses.

Light: Science \& Applications (2015) 4, e253; doi:10.1038/Isa.2015.26; published online 27 February 2015

Keywords: liquid crystal; porous graphene; terahertz; waveplate

\section{INTRODUCTION}

Terahertz (THz) waves, which are typically defined as electromagnetic waves in the frequency range of $0.1-10 \mathrm{THz}$, offer great potential for application in security screening, nondestructive evaluation and highspeed wireless communication. ${ }^{1,2}$ In the past two decades, impressive progress has been made in this field. ${ }^{1-4}$ It is expected that $\mathrm{THz}$ switches, attenuators, filters, polarization controllers and even routers will become available to handle $\mathrm{THz}$ waves in controllable or reconfigurable ways, ${ }^{5,6}$ just like their well-developed optical counterparts. Liquid crystals (LCs) have proven to be a perfect means of fulfilling these needs in the visible and telecom bands. It is also a very promising candidate for the new platform of $\mathrm{THz}$ devices. ${ }^{7,8}$ However, related studies still face significant challenges: First, metal layers with a thickness of more than a few tens of nanometers are totally opaque, and the conductive indium tin oxide (ITO) films that are commonly used in the visible range also become highly reflective. ${ }^{9}$ The lack of transparent electrodes makes the electric tuning of LCs difficult to achieve. Second, the dispersion of LC refractive indices induces a comparatively low birefringence in the THz regime. ${ }^{10}$ Third, both the corresponding wavelengths and wavelength range ( $30 \mu \mathrm{m}$ to $3 \mathrm{~mm}$ in vacuum) are much larger than those in the visible region. The latter two facts together give rise to a need for a very thick LC layer to achieve certain modulations (e.g., $\pi / 2$ or $\pi$ phase retardation), leading to several disadvantages such as high operating voltage, slow response and poor pre-alignment. These bottlenecks hinder the rapid development of this field.

In early studies, magnetic fields were used to tune LCs,${ }^{11,12}$ without the requisite transparent electrodes. These devices achieved good tunability, but they were bulky, heavy, costly and highly power consuming. Therefore, electrically driven devices remain the primary focus of research. Tsai et al. ${ }^{13}$ have employed a bias electric field generated by two crossed gold strips, with low modulation efficiency and high driving voltage. Hsieh et al. ${ }^{14}$ have accomplished greater modulation of up to $\pi / 2$ by using a lateral field to avoid affecting the transmittance of the THz signals, but this design suffered from a very slow response. We have used subwavelength metal wire grids as transparent electrodes for $\mathrm{THz}$ phase shifters, thereby achieving highly compact and efficient components. ${ }^{15}$ However, these electrodes were polarization selective. Most recently, highly transparent ITO nanowhisker electrodes, the transmittance of which reached $\sim 82 \%$ in the range of $0.2-$ 1.2 THz, have been utilized in THz phase shifters. ${ }^{16}$ Unfortunately, to achieve the required high phase retardation, in most previous efforts, large cell gaps (500 $\mu \mathrm{m}$ or more) have been introduced, causing the components to respond very slowly because the decay time of LCs is

${ }^{1}$ National Laboratory of Solid State Microstructures, Collaborative Innovation Center of Advanced Microstructures and College of Engineering and Applied Sciences, Nanjing University, Nanjing 210093, China; ${ }^{2}$ Research Institute of Superconductor Electronics (RISE), School of Electronic Science and Engineering, Nanjing University, Nanjing 210093 , China; ${ }^{3}$ State Key Laboratory of Materials Oriented Chemical Engineering, College of Materials Science and Engineering, Nanjing Tech University, Nanjing 210009 , China and

${ }^{4}$ Department of Chemistry, Tsinghua University, Beijing 100084, China

*These authors contributed equally to this work.

Correspondence: W Hu, College of Engineering and Applied Sciences, Nanjing University, Nanjing 210093, China

E-mail: huwei@nju.edu.cn

YQ Lu, College of Engineering and Applied Sciences, Nanjing University, Nanjing 210093, China

E-mail: yqlu@nju.edu.cn

Received 22 September 2014; revised 10 December 2014; accepted 12 December 2014; accepted article preview online 18 December 2014 
proportional to the square of the cell gap. ${ }^{17}$ In addition, it is quite difficult to implement homogeneous pre-alignment in such thick cells, as the coherence length of LCs that can be induced using an alignment layer is only $\sim 100 \mu \mathrm{m}$. This reduces the effective phase retardation and introduces scattering-induced loss. A stack-layered LC strategy has been utilized to overcome the coherence length limitation, ${ }^{12}$ but this strategy was found to incur additional loss. Meanwhile, new LC materials with greater birefringence have been successively reported. ${ }^{18-20}$ We have developed a room-temperature nematic LC NJU-LDn-4 with an average birefringence of greater than 0.3 from 0.5 to $2.5 \mathrm{THz}$, which is 2.5 times the birefringence of traditional E7 $(\sim 0.12$ in the THz region). ${ }^{21}$ This material improvement remarkably reduces the required cell gap, thus decreasing the operating voltage and response time.

In the past 10 years, graphene has attracted considerable attention because of its excellent electrical and optical performance. ${ }^{22}$ This promising material has also been applied in the THz region. ${ }^{23,24} \mathrm{Wu}$ et al..$^{25}$ have used pristine graphene films grown via chemical vapor deposition (CVD) as transparent electrodes to drive LCs and obtained a $10.8^{\circ}$ phase shift at $1.0 \mathrm{THz}$. The transmittance of single-layer graphene was found to be $88 \%$ in the $\mathrm{THz}$ range. It decreased gradually as the number of graphene layers was increased, whereas the electrical conductivity increased, implying a tradeoff between them. Notably, the growth and treatment of large-area single-layer graphene films are more challenging than those of few-layer ones. Here, porous few-layer graphene films are generated via a simple UV/ozone (UVO) technique, and their frequency-dependent transmittances are characterized. They exhibit much higher $\mathrm{THz}$ transparency $(>98 \%)$ than does singlelayer graphene while maintaining high electrical conductivity. A subwavelength metal wire grid is introduced to serve as the other electrode and built-in polarizer. By combining these elements with a high-birefringence NJU-LDn-4 LCs, a tunable THz half-wave plate over $1.0 \mathrm{THz}$ is obtained. The polarization evolution of the waveplate is directly demonstrated. The results suggest a practical design for various tunable $\mathrm{THz}$ devices.

\section{MATERIALS AND METHODS}

\section{Schematic and principle}

The cell configuration of the component is illustrated in Figure 1a. It is composed of two parallel fused silica substrates separated by $250-\mu \mathrm{m}$ thick Mylar films and infiltrated with the high-birefringence LCs. The front substrate (nearer the $\mathrm{THz}$ source) is covered with a metal wire grid, whereas the rear one is covered with porous few-layer graphene. Both substrates are spin coated with sulfonic azo dye (SD1) as an alignment layer and photo-aligned to achieve homogeneous prealignment at $45^{\circ}$ to the grating direction.

The principle applied here is quite similar to that of LC waveplates in the visible range. $\mathrm{THz}$ waves are linearly polarized by the sub-wavelength metal wire grid. Because the initial LC alignment is $45^{\circ}$ to the grid orientation, that is, $-45^{\circ}$ to the incident polarization, the linearly polarized wave is decomposed into two components: parallel and perpendicular to the LC director (the optical axis). Because of the birefringence of the LCs $\left(\Delta n, \Delta n=n_{\mathrm{e}}-n_{\mathrm{o}}, n_{\mathrm{e}}\right.$ - extraordinary refractive

a
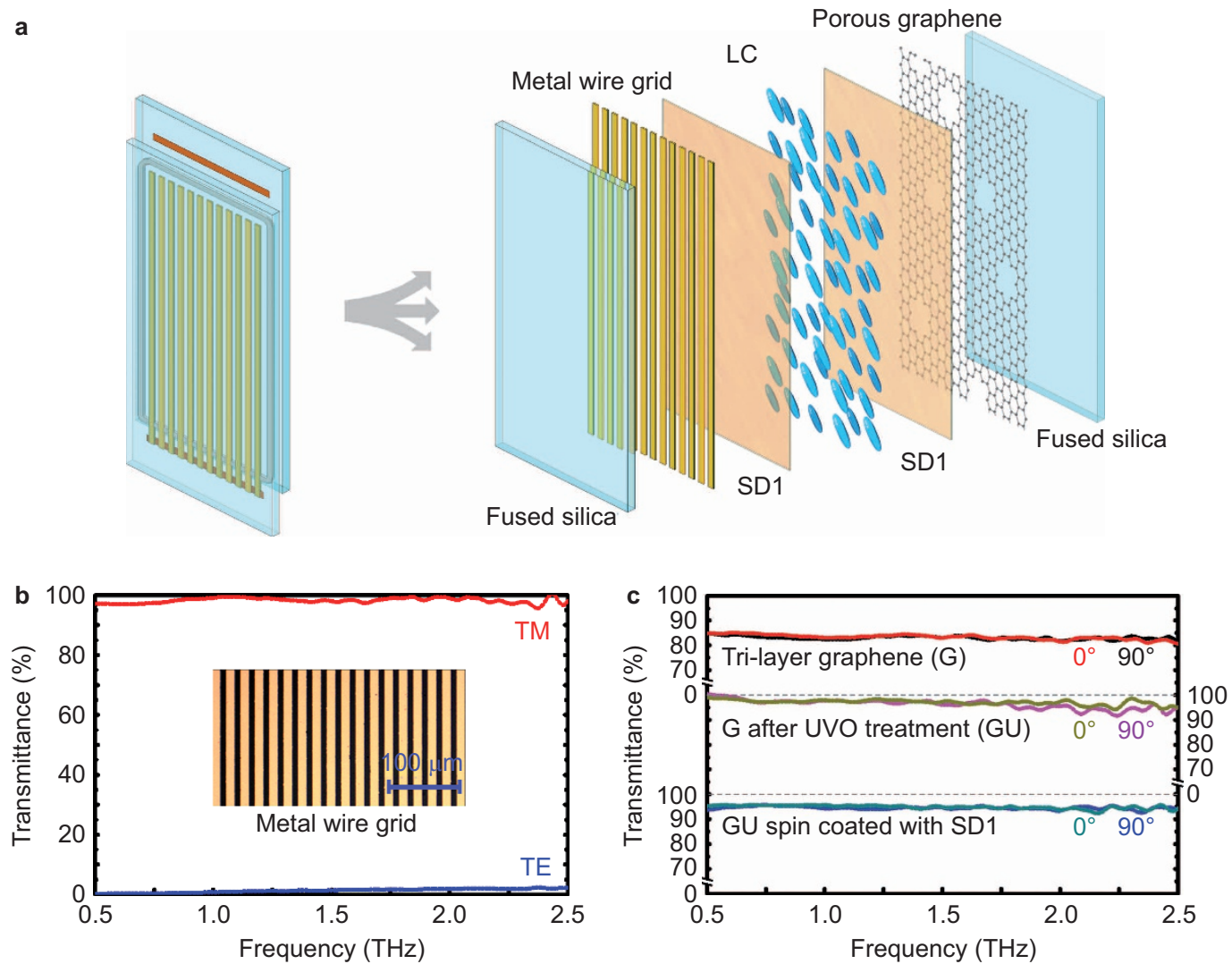

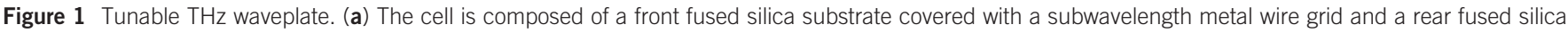

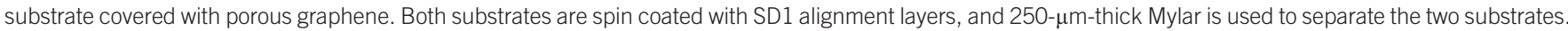

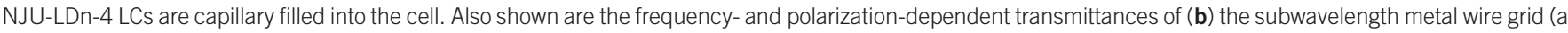

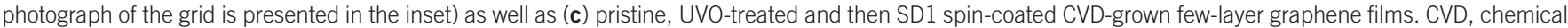
vapor deposition; LC, liquid crystal; SD1, sulfonic azo dye; TE, transverse electric; THz, terahertz; TM, transverse magnetic; UVO, UV/ozone. 
index; $n_{\mathrm{o}}$ - ordinary refractive index), the phase retardation between the ordinary and extraordinary components of the THz beam passing through this cell is $\Delta \varphi=2 \pi \Delta n d / \lambda$, where $d$ is the cell gap and $\lambda$ is the incident wavelength in vacuum. The highest phase retardation is achieved when the voltage is zero. As the applied voltage increases, $n_{\mathrm{o}}$ remains constant, while the effective value of $n_{\mathrm{e}}\left(n_{\mathrm{eff}}\right)$ decreases, thus allowing for the tuning of $\Delta \varphi$.

\section{Fabrication}

We first transferred the CVD-grown graphene onto the fused silica substrate as described in the literature. ${ }^{26}$ Then, the graphene film was UVO treated using a UV cleaning machine (FD/UVO3-05s; Fanda Machine Co., Suzhou, China) for $30 \mathrm{~min}$. This simple process introduced porous structures into the graphene films. The subwavelength metal wire grid was fabricated via photolithography and Au deposition, with a thickness of approximately $40 \mathrm{~nm}$, onto $0.6-\mathrm{mm}$-thick fused silica. ${ }^{15}$ The grating pitch was $20 \mu \mathrm{m}$ with a duty cycle of $50 \%$. Photo-sensitive SD1 (Dainippon Ink and Chemicals Inc., Chiba, Japan $)^{27}$ was dissolved in N,N-dimethylformamide (DMF) at a concentration of $0.5 \mathrm{wt}-\%$. The solution was spin coated onto the substrates coated with the UVO-treated graphene and the sub-wavelength metal wire grid at 3000 r.p.m. for $30 \mathrm{~s}$ to form the alignment layers. Then, the films were baked at $100{ }^{\circ} \mathrm{C}$ for $10 \mathrm{~min}$ to remove residual DMF. The two substrates $(1.5 \mathrm{~cm} \times 2.5 \mathrm{~cm}$ in size $)$ were assembled using epoxy glue and uniformly separated by $250-\mu \mathrm{m}$-thick Mylar films to form a cell. An exposure of $3 \mathrm{~J} \mathrm{~cm}^{-2}$ under a linearly polarized $405 \mathrm{~nm}$ blue LED was applied through the porous graphene substrate to induce uniform planar alignment in the cell. As the light was absorbed, the chromophores of the SD1 tended to rotate perpendicularly in the azimuth direction with respect to the direction of incident polarization. The LC pre-alignment was set at $45^{\circ}$ to the direction of the metal grid. Finally, the cell was infiltrated with NJU-LDn-4 LCs, which were prepared as previously reported..$^{21}$ To fabricate a doublestacked cell, an additional substrate covered with porous graphene films on both sides was inserted. The cells were driven by applying a $1 \mathrm{kHz}$ square-wave alternating current signal through copper tapes connecting the electrodes to the electric power source.

\section{Characterization}

The frequency- and polarization-dependent transmittances of the two different electrodes were characterized using a THz time-domain spectroscopy system (TAS7500SP; Advantest Corporation, Tokyo, Japan) with a transmission analysis accessory. Figure $1 \mathrm{~b}$ presents the transverse magnetic mode and transverse electric mode transmittances of the subwavelength metal wire grid, which was composed of alternating $10 \mu \mathrm{m}$ metal strips and $10 \mu \mathrm{m}$ spaces (as shown in the inset). The metal wire grid exhibited a high transmittance of $\sim 98 \%$ on average in the range of $0.5-2.5 \mathrm{THz}$ for transverse magnetic waves. Meanwhile, the transverse electric waves were highly suppressed, and a transmittance below $2 \%$ was obtained. Thus, the grid served as both a transparent electrode and a built-in broadband polarizer. ${ }^{15}$ Figure $1 \mathrm{c}$ reveals that the few-layer (trilayer, on average) CVD graphene exhibited moderate transparency $(\sim 82 \%)$ in the $\mathrm{THz}$ band. After the single-step UVO treatment, the transmittance of the few-layer graphene increased to over $98 \%$, even higher than that of single-layer graphene. ${ }^{25}$ A superb transparency greater than $95 \%$ was still achieved after SD1 spin coating. All transmittances reported here are referred to the same fused silica substrate without graphene coating. Furthermore, all graphene films mentioned above exhibited excellent polarization-independent transmittance in the tested $\mathrm{THz}$ range. Both the transmittance of the few-layer graphene at $\mathrm{THz}$ frequencies and its sheet resistance are important factors. The sheet resistance of the UVO-treated graphene was $\sim 910 \Omega \mathrm{sq}^{-1}$, as measured using a four-point probe instrument. This value is comparable to that of a pristine single-layer graphene film. Although this result is acceptable for single-pixel applications, further improvement is still desired, especially for high-resolution devices. The value of the sheet resistance is quite sensitive to film quality and could be improved by optimizing the CVD process. However, similar to the case of ITO, there is always a tradeoff between resistivity and transmittance. Moreover, in this study, a photo-alignment technique ${ }^{27,28}$ was applied to achieve precise and stable homogeneous alignment and to avoid any mechanical damage to either of the frail electrodes.

Morphological characterization and Raman spectroscopy analysis were performed to determine the reason for the pronounced improvement in the transparency of the graphene films observed after UVO treatment. A pristine CVD-grown graphene film was transferred onto a microhollow carbon-coated copper grid for transmission electron microscopy (TEM) inspection. The edges of the suspended film tended to fold back, allowing for a cross-sectional view of the film, which provided an accurate means of measuring the number of layers. ${ }^{29}$ The darker region visible in the center of Figure 2 a was caused by the doubling of the layers at the folded edge. Sections of trilayers are observed in Figure 2b. The estimated interlayer spacing was $3.3 \AA$. Figure 2e presents the corresponding Raman spectrum, which allowed for structural and quality characterization of the graphene films. Three peaks are evident for the pristine graphene, where $\mathrm{G}\left(\sim 1580 \mathrm{~cm}^{-1}\right)$ denotes the symmetry-allowed graphite band, $\mathrm{D}\left(\sim 1350 \mathrm{~cm}^{-1}\right)$ is disorder-induced and 2D $\left(\sim 2700 \mathrm{~cm}^{-1}\right)$ is the second harmonic band of D. The G-to-2D peak intensity ratio exhibits a good correlation with the number of graphene layers in CVD graphene samples. ${ }^{29}$ The spectrum presented in Figure 2e is indicative of few-layer graphene, which is consistent with our TEM measurement. Scanning electron microscope observation (Figure 2c) revealed that the CVD-grown graphene films were solid and smooth. After UVO treatment, the graphene was oxidized, carbon-carbon bonds were broken and $\mathrm{CO} / \mathrm{CO}_{2}$ molecules were released; ${ }^{30}$ thus, randomly distributed microscale hollows appeared (Figure 2d). Because the spot size for Raman excitation is at the microscale, the recorded spectra were highly location dependent. Two typical spectra are presented in Figure 2f. The strong and sharp D band in the spectrum indicated by the red line indicates that more defects appeared after UVO treatment, whereas the decreased G/ $2 \mathrm{D}$ ratio indicated by the black line suggests a reduction in the graphene film layers. These phenomena are both consistent with the scanning electron microscope observations. The porous structure induced by the perforation effect of the UVO treatment is considered to be the reason for the observed increase in transmittance.

\section{RESULTS AND DISCUSSION}

\section{Cell simulations}

Here, we use a 3D module of the commercial software Techwiz LCD to simulate the electrical field and director distribution in the cell. As in the experiment, a $1 \mathrm{kHz}$ square-wave alternating current signal is used. The top electrode contains alternating $10 \mu \mathrm{m}$ metal strips and $10 \mu \mathrm{m}$ spaces. The bottom electrode (porous graphene) is equivalently replaced by a flat ITO electrode with micro-sized hollows in the simulation. The $y$ axis is defined as the direction of the metal wire grid, and the $x$ axis is perpendicular to the $y$ axis in the plane of the substrates, whereas the $z$ axis is normal to the substrates. Only the electrical field along the $z$ axis is shown, and its intensity scale is defined by a color bar. Figure $3 \mathrm{a}$ and $3 \mathrm{c}$ shows the electrical field and LC director distribution 

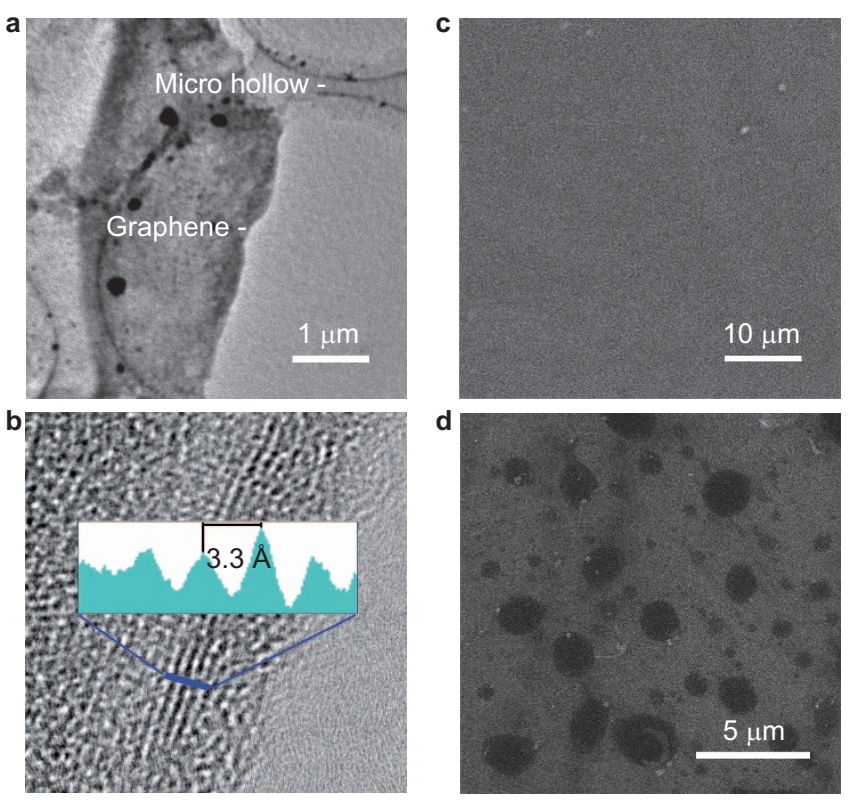
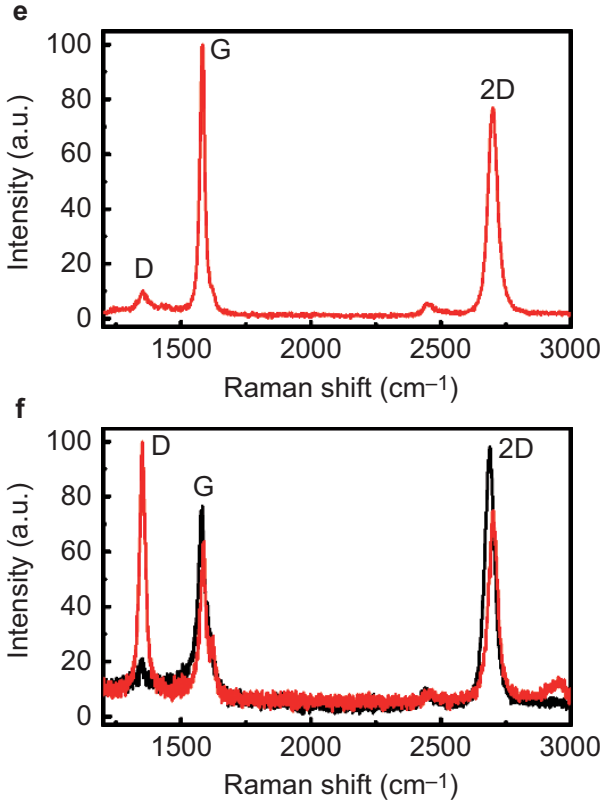

Figure 2 Graphene films. (a) Low-magnification TEM image showing a CVD-grown graphene film on a microhollow carbon-coated grid. (b) High-magnification TEM image showing the film edge, consisting of trilayer graphene. A cross-sectional analysis is presented as an inset. SEM images of (c) pristine and (d) UVO-treated graphene films. Raman spectra of (e) pristine and (f) UVO-treated graphene films; the black line indicates the reduction of the graphene film layers, whereas the red line indicates the appearance of more defects. CVD, chemical vapor deposition; SEM, scanning electron microscope; TEM, transmission electron microscopy; UVO, UV/ozone.

at $0 \mathrm{~V}_{\mathrm{rms}}$, respectively. The field intensity is $0 \mathrm{~V} \mu \mathrm{m}^{-1}$, and the LC director is homogenously oriented at $45^{\circ}$ to the $y$ axis. The LC director is reoriented by the electric field as the applied voltage increases. Figure $3 \mathrm{~b}$ shows the field distribution inside the cell at $50 \mathrm{~V}_{\mathrm{rms}}$, which is approximately $0.2 \mathrm{~V} \mu \mathrm{m}^{-1}$, as expected. Figure $3 \mathrm{~d}$ shows the corresponding LC director distribution. All LCs, except for the two thin layers adjacent to the electrodes, reorient along the direction of the applied field. The simulation results indicate that because the cell gap is much larger than the pitch of the metal grid and the size of the graphene hollows, a uniform electric field and homogeneous rotation of the LC director can be achieved, which further confirms the operation of the device as a tunable waveplate.

\section{Electro-optical properties}

A THz time-domain spectroscopy system with a transmission polarization analysis accessory was used to characterize the properties of proposed waveplate. Figure 4a schematically illustrates the setup for the transmittance polarization analysis. Figure $4 \mathrm{~b}$ presents the voltagedependent temporal spectra of ordinary and extraordinary $\mathrm{THz}$ waves, respectively. The spectra of the ordinary waves did not change with the applied voltage, whereas those of the extraordinary waves shifted considerably. We transformed the temporal spectra into frequency spectra using fast Fourier transforms. Figure $4 \mathrm{c}$ shows the frequency-dependent phase retardation at several different operating voltages, as extracted from the frequency spectra. By virtue of the high birefringence of the NJU-LDn-4 LCs, significant phase retardation was achieved. When values of $\Delta n=0.3$ and $d=250 \mu \mathrm{m}$ are considered in $\Delta \varphi=2 \pi \Delta n d / \lambda$, it is evident that a theoretical $\Delta \varphi$ of $\pi$ could be achieved at $2.0 \mathrm{THz}$. In our experiments, a half-wave plate was realized at $2.1 \mathrm{THz}$ when no field was applied. The retardation at a given voltage was nearly directly proportional to the frequency because of the low dispersion of the LC birefringence over a broadband range. As

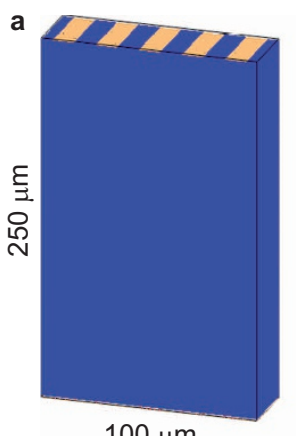

$100 \mu \mathrm{m}$
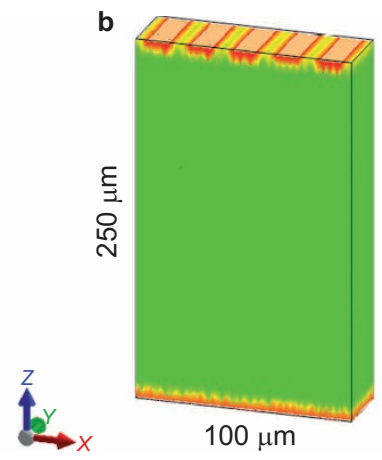
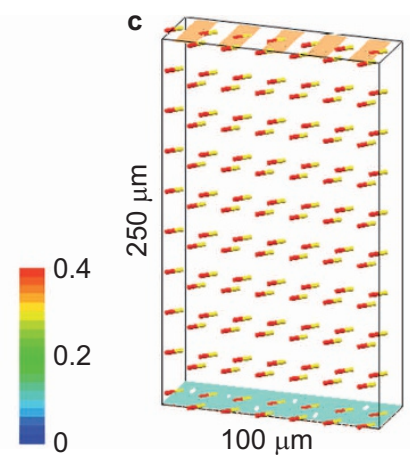

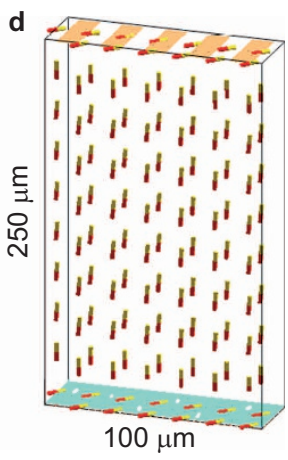

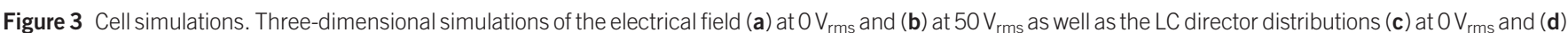

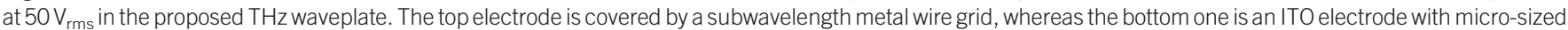

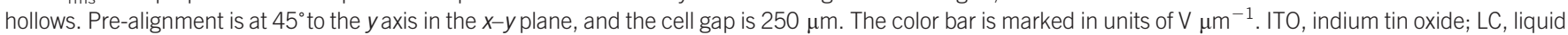
crystal; THz, terahertz. 

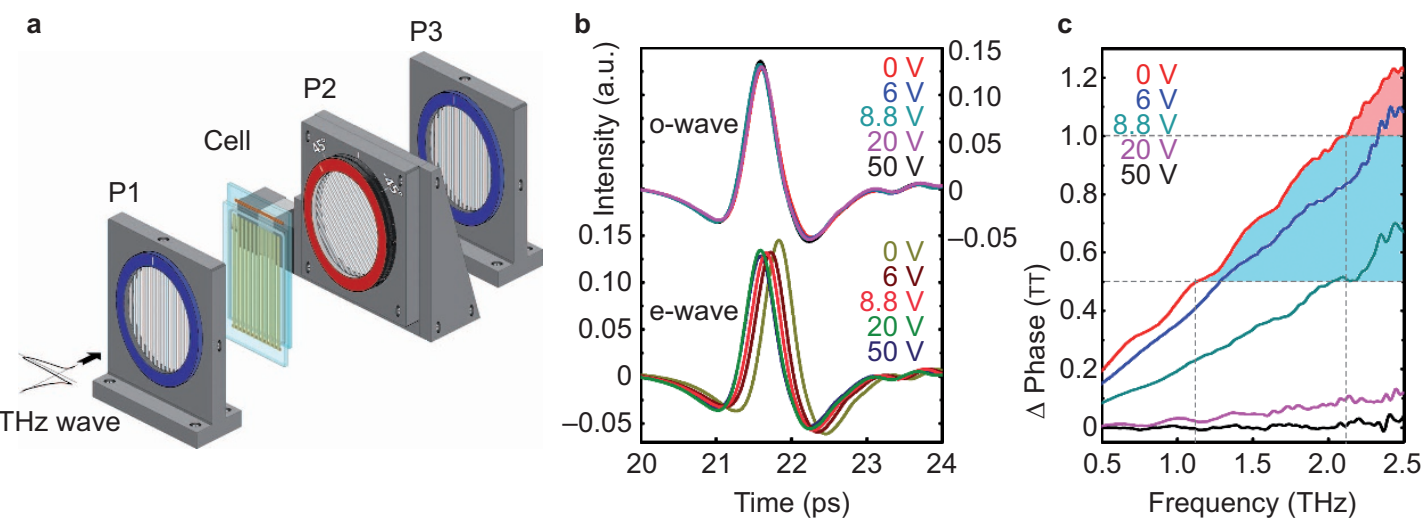

d
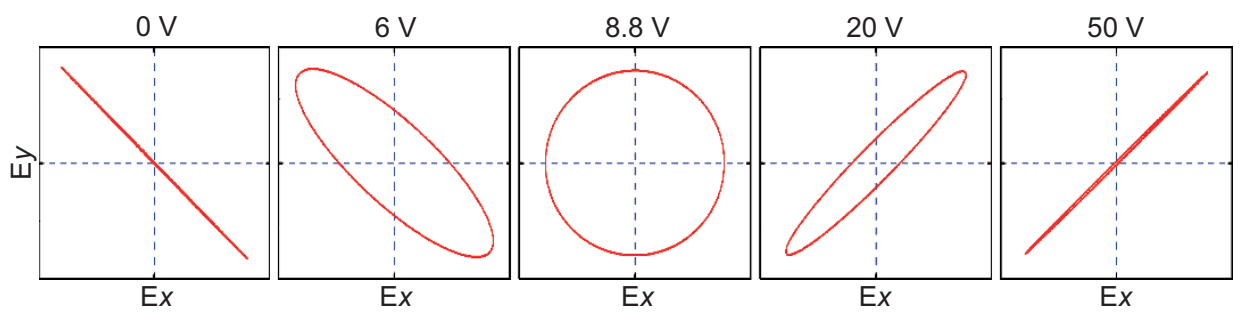

Figure 4 Device characteristics. (a) Schematic set-up for transmittance polarization analysis. The apparatus consists of three metal wire grid polarizers (P), where P1 and P3 are fixed in a parallel orientation (defined as $0^{\circ}$ ), thereby allowing only TM-mode waves to be transmitted to the cell, whereas P2 can rotate between the $45^{\circ}$ and $45^{\circ}$ states. (b) Voltage-dependent temporal spectra of ordinary and extraordinary THz waves transmitted through the cell. (c) Frequency-dependent phase retardation calculated from the curves presented in $\mathbf{b}$ for different operating voltages. (d) Polarization evolution at 2.1 THz: linearly polarized at 0 V, elliptically polarized at 6 V, circularly polarized at $8.8 \mathrm{~V}$, elliptically polarized at $20 \mathrm{~V}$ and linearly polarized at $50 \mathrm{~V}$ (orthogonal to the polarization at $0 \mathrm{~V}$ ). THz, terahertz; TM, transverse magnetic.

expected, the phase retardation decreased from its maximum to nearly zero as the applied voltage was progressively increased to saturation, $\sim 50 \mathrm{~V}_{\mathrm{rms}}$ in this case. For the tested sample, a $\Delta \varphi$ tuning of $0-\pi$ was achieved for frequencies above $2.1 \mathrm{THz}$ (labeled in red in Figure 4c), and a tuning of $0-\pi / 2$ was achieved above $1.1 \mathrm{THz}$ (both blue and red regions in Figure 4c). The polarization evolution at $2.1 \mathrm{THz}$ is illustrated in Figure 4d. The output polarization states changed from a linear polarization (half-wave plate) to elliptical, circular (quarterwave plate) and again elliptical polarizations and then back to an orthogonal linear polarization (no phase retardation) at increasing voltages.

\section{Double-stacked LC cell}

Greater modulation and a broader tunable range can be accomplished by stacking cells. We demonstrated a double-stacked waveplate with a built-in polarizer (as shown in Figure 5a). The cell configuration was quite similar to that of the single cell (Figure 1a), except that an extra substrate covered with porous graphene films on both sides was inserted between the two initial substrates. In fact, graphene films, including porous ones, could replace the metal wire grid electrode both here and in other devices. ${ }^{31,32}$ The effective LC thickness was nearly doubled, thereby allowing the device to function as a quarterwave plate over the entire testing range $(0.5-2.5 \mathrm{THz})$. A $\Delta \varphi$ tuning of $0-\pi$ for frequencies above $0.9 \mathrm{THz}$ (red and yellow regions in Figure $5 b$ ) and even a tuning of over $2 \pi$ for frequencies above $1.8 \mathrm{THz}$ (yellow regions in Figure 5b) were achieved. Therefore, arbitrary polarization conversion could be performed above $1.8 \mathrm{THz}$ using the sample by tuning the applied voltage. The voltage-dependent phase retardation measured at 0.9 and $1.8 \mathrm{THz}$ is presented in Figure $5 \mathrm{c}$. As expected, the phase retardation decreased as the applied
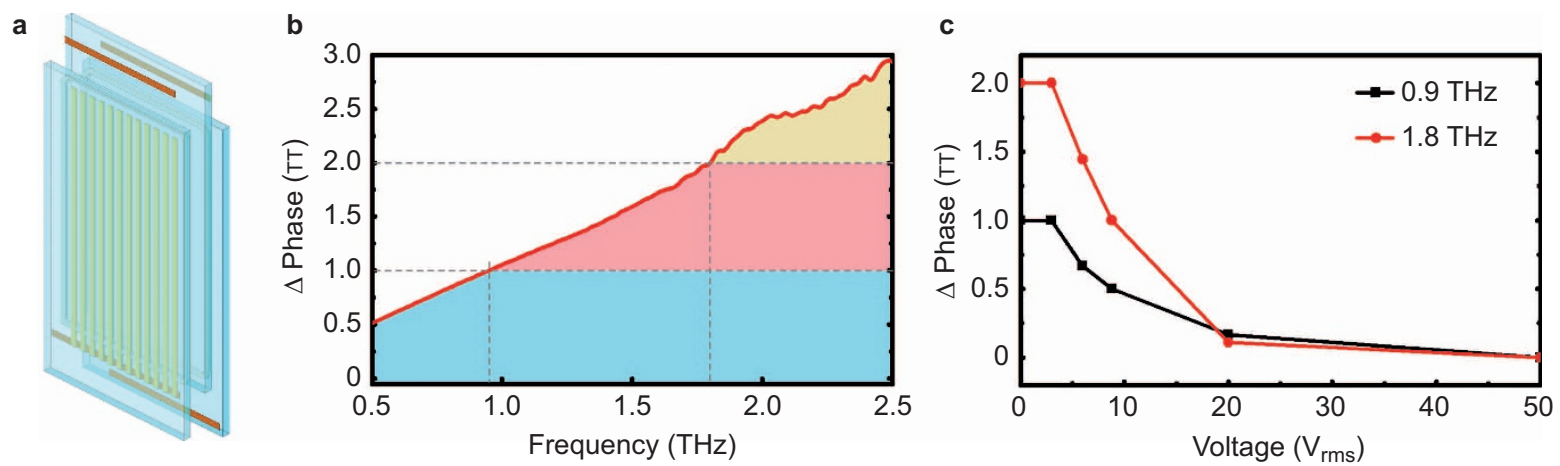

Figure 5 Double-stacked cell characterization. (a) Schematic illustration of the double-stacked cell. (b) Frequency-dependent phase retardation of the sample at 0 $\mathrm{V}_{\text {rms. }}$ (c) Voltage-dependent phase retardation at 0.9 and $1.8 \mathrm{THz}$. THz, terahertz. 
voltage increased. A tuning range of greater than $\pi$ was achieved at 1.8 THz below $9 \mathrm{~V}_{\mathrm{rms}}$. Because the loss of the components was highly suppressed, the cell-stacking strategy became much more powerful.

\section{CONCLUSION}

In this work, we systematically solved the problems encountered in the design of LC-based $\mathrm{THz}$ components. A practical, tunable $\mathrm{THz}$ waveplate, which is a fundamental element for polarization conversion, ${ }^{33}$ was proposed and demonstrated. In addition to its compact size, high efficiency and low power consumption, it also offers other interesting features and advantages: (i) its unique cell structure contains a built-in subwavelength metal wire grid polarizer/electrode and a cost-effective CVD-grown few-layer graphene electrode; (ii) a large-area UVO posttreatment technique is applied to significantly improve the transparency of the graphene in the $\mathrm{THz}$ band; (iii) a non-contact photo-alignment technique is employed to implement precise and high-quality LC prealignment on the frail electrodes; (iv) a high-birefringence LC mixture is used, enabling high phase retardation in a single cell; and (v) continuous tuning of polarization conversion is possible at low driving voltages $\left(0-50 \mathrm{~V}_{\mathrm{rms}}\right)$. The same tuning range can be achieved below 10 $\mathrm{V}_{\text {rms }}$ using a double-stacked LC cell. Because a total solution for a half-wave plate with a similar design in the visible range has been demonstrated, the development of various high-performance $\mathrm{THz}$ photonic devices for further amplitude, phase, and polarization modulation and even complex wavefront control, such as Q-plates, ${ }^{34}$ fork gratings, ${ }^{35}$ etc., can also be expected. We believe that this total solution may pave a simple and bright road toward the development of various tunable $\mathrm{LC} \mathrm{THz}$ apparatuses, which will play important roles in $\mathrm{THz}$ imaging, sensing, high-speed wireless communication and security applications.

\section{ACKNOWLEDGEMENTS}

The authors are indebted to V Chigrinov for his kind support with the photoalignment technique and would like to thank Wen-Chao Liu and Li-Gang Ma for their assistance with the Raman spectroscopy measurements. This work was sponsored by the 973 programs (Nos. 2011CBA00200 and 2012CB921803), the National Natural Science Foundation of China (Nos. 61490714, 11304151 and 61435008) and the PhD Programs Foundation of the Ministry of Education of China (No. 20120091120020). Yan-Qing Lu appreciates the support from National Science Fund for Distinguished Young Scholars (No. 61225026) and the Program for Changjiang Scholars and Innovative Research Team in University (No. IRT13021). Biao-Bing Jin appreciates the support from the 973 program (No. 2014CB339800), the National Natural Science Foundation of China (Nos. 61371035 and 11227904) and Cooperative Innovation Centre of Terahertz Science, University of Electronic Science and Technology (Chengdu, China).

1 Zhang XC, Xu JZ. Introduction to THz Wave Photonics. New York: Springer; 2010

2 Koenig S, Lopez-Diaz D, Antes J, Boes F, Henneberger R et al. Wireless sub-THz communication system with high data rate. Nat Photonics 2013; 7: 977-981.

3 Ferguson B, Zhang XC. Materials for terahertz science and technology. Nat Mater 2002; $1: 26-33$.

4 Tonouchi M. Cutting-edge terahertz technology. Nat Photonics 2007; 1: 97-105.

5 Kamaraju N, Rubano A, Jian L, Saha S, Venkatesan T et al. Subcycle control of terahertz waveform polarization using all-optically induced transient metamaterials. Light Sci Appl 2014; 3: e155; doi:10.1038/lsa.2014.36.
$6 \quad$ Ma FS, Lin YS, Zhang X, Lee C. Tunable multiband terahertz metamaterials using a reconfigurable electric split-ring resonator array. Light Sci App/ 2014; 3: e171; doi: $10.1038 /$ lsa.2014.52.

7 Wu ST, Efron U, Hess LD. Infrared birefringence of liquid crystals. App/ Phys Lett 1984; 44: 1033-1035.

8 Wilk R, Vieweg N, Kopschinski O, Koch M. Liquid crystal based electrically switchable Bragg structure for $\mathrm{THz}$ waves. Opt Express 2009; 17:7377-7382.

9 Chen CW, Lin YC, Chang CH, Yu PC, Shieh JM et al. Frequency-dependent complex conductivities and dielectric responses of indium tin oxide thin films from the visible to the far-infrared. IEEE J Quantum Electron 2010; 46: 1746-1754.

10 Wu ST. Birefringence dispersions of liquid crystals. Phys Rev A 1986; 33: 1270 1274.

11 Chen CY, Tsai TR, Pan CL, Pan RP. Room temperature terahertz phase shifter based on magnetically controlled birefringence in liquid crystals. App/ Phys Lett 2003; 83: 4497-4499.

12 Chen CY, Pan CL, Hsieh CF, Lin YF, Pan RP. Liquid-crystal-based terahertz tunable Lyot filter. Appl Phys Lett 2006; 88: 101107.

13 Tsai TR, Chen CY, Pan RP, Pan CL, Zhang XC. Electrically controlled room temperature terahertz phase shifter with liquid crystal. IEEE Microwave Wireless Compont Lett 2004; 14: 77-79.

14 Hsieh CF, Pan RP, Tang TT, Chen HL, Pan CL. Voltage-controlled liquid-crysta terahertz phase shifter and quarter-wave plate. Opt Lett 2006; 31: 1112-1114.

15 Lin XW, Wu JB, Hu W, Zheng ZG, Wu ZJ et al. Self-polarizing terahertz liquid crystal phase shifter. AIP Adv 2011; 1: 032133.

16 Yang CS, Tang TT, Chen PH, Pan RP, Yu P et al. Voltage-controlled liquid-crystal terahertz phase shifter with indium-tin-oxide nanowhiskers as transparent electrodes. Opt Lett 2014; 39: 2511-2513.

17 Khoo IC, Wu ST. Optics and Nonlinear Optics of Liquid Crystals. Singapore: World Scientific; 1993.

18 Wiesauer $\mathrm{K}$, Jördens $\mathrm{C}$. Recent advances in birefringence studies at $\mathrm{THz}$ frequencies. J Infrared Milli Terahz Waves 2012; 34: 663-681.

19 Gauza S, Wu ST, Spadło A, Dabrowski R. High performance room temperature nematic liquid crystals based on laterally fluorinated isothiocyanato-tolanes. J Display Technol 2006; 2: 247-253.

20 Gauza S, Zhao Y, Cor TL, Wu ST, Dziaduszek J et al. Enhancing birefringence by doping fluorinated phenyltolanes. J Display Technol 2006; 2: 327-332.

21 Wang L, Lin XW, Liang X, Wu JB, Hu W et al. Large birefringence liquid crystal material in terahertz range. Opt Mater Express 2012; 2: 1314-1319.

22 Geim AK, Novoselov KS. The rise of graphene. Nat Mater 2007; 6: 183-191.

23 Vicarelli L, Vitiello M, Coquillat D, Lombardo A, Ferrari A et al. Graphene field-effect transistors as room-temperature terahertz detectors. Nat Mater 2012; 11: 865-871.

24 Ju L, Geng B, Horng J, Girit C, Martin M et al. Graphene plasmonics for tunable terahertz metamaterials. Nat Nanotechnol 2011; 6: 630-634.

25 Wu Y, Ruan X, Chen CH, Shin YJ, Lee Y et al. Graphene/liquid crystal based terahertz phase shifters. Opt Express 2013; 21: 21395-21402.

26 Li X, Zhu Y, Cai W, Borysiak M, Han B et al. Transfer of large-area graphene films for high-performance transparent conductive electrodes. Nano Lett 2009; 9: 43594363.

27 Akiyama H, Kawara T, Takada H, Takatsu H, Chigrinov V et al. Synthesis and properties of azo dye aligning layers for liquid crystal cells. Liq Cryst 2002; 29: 1321-1327.

28 Schadt M, Seiberle H, Schuster A. Optical patterning of multi-domain liquid-crystal displays with wide viewing angles. Nature 1996; 381: 212-215.

29 Reina A, Jia X, Ho J, Nezich D, Son $\mathrm{H}$ et al. Large area, few-layer graphene films on arbitrary substrates by chemical vapor deposition. Nano Lett 2008; 9: 30-35.

30 Yang DP, Wang X, Guo X, Zhi X, Wang K et al. UV/O ${ }_{3}$ Generated graphene nanomesh: formation mechanism, properties, and FET studies. J Phys Chem C 2014; 118: 725731.

31 Cong LQ, Xu NN, Gu JQ, Singh RJ, Han JG et al. Highly flexible broadband terahertz metamaterial quarter-wave plate. Laser Photonics Rev 2014; 8: 626-632.

32 Chen ZX, Chen JH, Wu ZJ, Hu W, Zhang XJ et al. Tunable Fano resonance in hybrid graphene-metal gratings. Appl Phys Lett 2014; 104: 161114.

33 Kawada Y, Yasuda T, Nakanishi A, Akiyama K, Hakamata K et al. Achromatic prismtype wave plate for broadband terahertz pulses. Opt Lett 2014; 39: 2794-2797.

34 Slussarenko S, Murauski A, Du T, Chigrinov V, Marrucci L et al. Tunable liquid crystal q-plates with arbitrary topological charge. Opt express 2011; 19: 4085-4090.

35 Wei BY, Hu W, Ming Y, Xu F, Rubin S et al. Generating switchable and reconfigurable optical vortices via photopatterning of liquid crystals. Adv Mater 2014; 26:15901595.

This work is licensed under a Creative Commons Attribution 3.0 Unported License. The images or other third party material in this article are included in the article's Creative Commons license, unless indicated otherwise in the credit line; if the material is not included under the Creative Commons license, users will need to obtain permission from the license holder to reproduce the material. To view a copy of this license, visit http://creativecommons.org/licenses/by/3.0/ 